\title{
Migrantes conectados. El impacto de la telefonía móvil en la migración
}

Migrants connectés. L'impact de la téléphonie mobile dans la migration

Connected migrants. The impact of wireless telephony on migration

\section{Daniela Castro Cabalceta}

\section{OpenEdition}

\section{Journals}

Edición electrónica

URL: http://journals.openedition.org/ctd/539

DOI: $10.4000 /$ ctd.539

ISSN: 2491-1437

Editor

Chaire Unesco Pratiques émergentes en technologies et communication pour le développement

Referencia electrónica

Daniela Castro Cabalceta, « Migrantes conectados. El impacto de la telefonía móvil en la migración », Communication, technologies et développement [En línea], 6 | 2018, Publicado el 18 diciembre 2018, consultado el 19 abril 2019. URL : http://journals.openedition.org/ctd/539; DOI : 10.4000/ctd.539

Este documento fue generado automáticamente el 19 abril 2019.

Communication, technologies et développement 


\title{
Migrantes conectados. El impacto de la telefonía móvil en la migración
}

\author{
Migrants connectés. L'impact de la téléphonie mobile dans la migration \\ Connected migrants. The impact of wireless telephony on migration
}

Daniela Castro Cabalceta

\section{Introducción}

1 Uno de los fenómenos que más caracteriza la época actual, y la diferencia del resto, es la intensificación de los movimientos migratorios transnacionales, la cual ha generado cambios importantes en la geopolítica mundial. Ante la expansión de los flujos migratorios a nivel planetario vemos como los cierres de las fronteras de los países del hemisferio norte no han detenido los movimientos de personas, sino que estos mismos se han redireccionado hacia otros destinos y en este punto resulta notorio el incremento de los flujos migratorios interregionales sur sur.

2 La ruta migratoria en Centroamérica es una de las más importantes del mundo, se estima que abarca un $3 \%$ de las migraciones mundiales y para América Latina este porcentaje aumenta a $6 \%$. En Centroamérica se estima que $12 \%$ de la población no vive en su país de nacimiento. Estos datos son el resultado de una investigación realizada por Carlos Sandoval García ${ }^{1}$, docente e investigador de la Universidad de Costa Rica. El autor también sostiene que "en Centroamérica la migración internacional duplica y cuadruplica las estimaciones regionales e internacionales".

3 A pesar de que la migración de personas de Centroamérica hacia Estados Unidos es la principal dinámica migratoria en la región, la migración extra regional se ha incrementado de forma importante su flujo en el corredor centroamericano. Datos del Instituto Nacional de Migración de México $^{2}$ señalan que en 2008 las autoridades mexicanas detuvieron a 326 asiáticos y 658 africanos y que para 2015 ese número había aumentado a 2,224 asiáticos y 2,078 africanos. Es decir, se registró un crecimiento de $679 \%$ y de $311 \%$ respectivamente. 
4 La migración contemporánea tiene como característica principal la irrupción de las nuevas tecnologías de la información y comunicación (NTICS) en todas sus dimensiones. Desde el comienzo de la preparación del viaje hasta el momento de integración en las sociedades de acogida, el teléfono móvil se ha vuelto una herramienta indispensable para el migrante. En base a esto vemos como los migrantes logran mantener de manera casi diaria y a distancia, el contacto con sus relaciones más cercanas sin importar las geografías, en un tipo de relación que se asemeja a la relación de proximidad. El vínculo "virtual", ya sea por medio del teléfono o el email, permite de manera cada vez más frecuente, la posibilidad de seguir estando presente en la familia y en las relaciones de los países de origen. La imagen del migrante sin raíces, como la figura paradigmática del mundo de los migrantes, se aleja cada vez más de la realidad para dar lugar a una figura, todavía mal definida, de un migrante que se desplaza constantemente y en sus andares cuenta con alianzas estratégicas que va desarrollando a lo largo del camino.

5 Tenemos acá movimientos cada vez más complejos y diversos que poco tienen que ver con las migraciones conocidas otrora, dónde nos interesa particularmente estudiar de qué manera las nuevas tecnologías de la comunicación están reconfigurando la vivencia de la migración.

6 ¿Podemos afirmar que el teléfono móvil está dotando al migrante de capacidades individuales para transformar su realidad y poder dar paso al desarrollo de tácticas inéditas en temas de movilidad, integración en las sociedades de acogida, así como estrategias de sobrevivencia comunitaria?

7 Para responder a estas interrogantes nos basamos en una observación no participante y en encuestas de tipo semi-directivas. El primer terreno en el que llevamos a cabo nuestro trabajo de campo fue durante varios meses en los centros de atención para migrantes haitianos en Costa Rica ubicados en la zona norte del país, frontera con Nicaragua. En este tiempo pudimos llevar a cabo al menos 150 entrevistas semi-directivas con los migrantes, hombres y mujeres, con edades entre los 20 y 40 años. Seguidamente continuamos trabajando con una población más reducida de migrantes, aquellos que decidieron instalarse en el país y han ido desarrollando estrategias de inserción en sus sociedades de acogida. En ambos terrenos de estudio nos interesó trabajar el tema de la telefonía móvil y sus usos por parte de esta población en sus experiencias migratorias.

8 Nuestro trabajo se encuentra en el cruce de varas áreas de estudio (nos interesan los desarrollos tecnológicos, así como la vivencia de la migración) que se conjugan en la interdisciplinariedad para poder estudiar la dimensión del uso de la NTICS por un grupo predeterminado de personas.

9 En una primera parte contextualizaremos el caso particular de la migración haitiana en el continente americano y el papel de Costa Rica como destino "de paso" en estos trayectos. Una vez contextualizado el fenómeno, analizaremos algunas posturas teóricas que han estudiado los flujos migratorios para subrayar la necesidad de sobrepasar estos análisis y proponer posibles pistas para entender las nuevas configuraciones de la migración interregional. Por último, analizaremos en detalle un ejemplo de cómo el teléfono móvil permitió a los migrantes haitianos desarrollar capacidades propias que se transformaron en estrategias innovadoras en los procesos de articulación transfronterizos. De igual modo veremos cómo por medio del móvil se crean flujos importantes de intercambios globales que participan en la consolidación de comunidades trasnacionales que permiten pensar la mundialización (así como la tecnología) desde su lado humano, es decir, dejar de 
lado las perspectivas puramente económicas para pensarla también como un movimiento de personas a través del planeta, con todas las implicaciones que esto puede tener en la reconfiguración de los patrones migratorios.

\section{La migración haitiana en la ruta migratoria centroamericana}

\section{Los desplazamientos de la población haitiana a través de los tiempos}

10 La migración haitiana tiene una larga historia, desde finales del siglo XIX y principios del siglo XX un número importante de haitianos empezaron a salir del país rumbo a Cuba y a República Dominicana para servir de mano de obra en las plantaciones de caña de azúcar. Esta migración fue de tipo "temporal” y fue hasta la crisis de los años 30 en que empezó la migración a gran escala con destinos como lo son los Estados Unidos, Canadá y Francia. A partir de la década de los 60's la migración se vio intensificada y se extendió como una práctica cultural en la sociedad haitiana (Nieto, 2014 : 14). Según los datos del Ministerio de los Haitianos viviendo en el Extranjero, creado en el año 1995 bajo el gobierno de JeanBertrand Aristide, para el año 2003 Haití tenía una población de dos millones de migrantes para una población total de ocho millones.

11 Cuando el terremoto de 7,3 grados en la escala de Richter sacudió a Haití el 12 de enero de 2010, los desplazados, forzados tanto dentro como fuera de la isla, se incrementaron considerablemente y tuvo como resultado lo que ha sido considerado como una de las mayores catástrofes urbanísticas y humanitarias del siglo XXI. Esta situación generó una nueva oleada de desplazados haitianos a lo largo del continente americano con una diversificación de los destinos, incluyendo países como Brasil y Chile. Cabe señalar que luego del terremoto, en los Estados Unidos se otorgó a las personas de nacionalidad haitiana en situación irregular el estatuto de Protección Temporal, el mismo estatuto que tiene las personas de nacionalidad cubana en dicho país.

12 El complejo escenario que presenta Haití nos hace pensar que inclusive la categoría de "migración" no es suficiente para comprender el alcance que tienen estos movimientos, dados los distintos tipos de violencia con los que estas personas se ven enfrentadas, que las obligan a desplazarse continuamente en busca de algún lugar donde puedan establecerse y llevar una mejor vida. La crisis humanitaria y estructural que se vive en Haití, así como el contexto global de desigualdades económicas y la mundialización de las migraciones generan constantemente gran cantidad de desplazados y es por eso por lo que desde hace algunos años empezaron a observarse en varias partes de Latinoamérica, grupos de personas haitianas buscando un lugar donde poder detener su marcha y empezar un nuevo proyecto de vida. En ese momento Brasil y Chile se posicionaron como nuevos destinos de referencia al tiempo que otros países como Perú, Panamá o Costa Rica se constituyeron como destinos de tránsito.

\section{El caso de la migración haitiana en territorio costarricense}

Como mencionamos anteriormente, Costa Rica se ha constituido como un punto de pasaje obligatorio en la ruta hacia Norte América y podemos considerar al país no como un país 
de destino sino más bien como un país de "tránsito". Sin embargo, la frontera entre Costa Rica y Nicaragua se convirtió en un gran nudo para los migrantes que siguen la ruta Centroamericana. En noviembre de 2015 se dio la llegada de miles de cubanos a la región en ruta hacia los Estados Unidos, ya que estaba latente la amenaza del fin de la política que permitía la permanencia de cubanos en los Estados Unidos, cuyo fin se vino a confirmar en enero de 2017 por el entonces presidente del país Barack Obama. En este momento, Nicaragua cerró sus fronteras, alegando crisis humanitaria, mientras Costa Rica mantuvo una política de puertas abiertas. Después de dos meses de estar varados en la frontera, el gobierno de Costa Rica facilitó la llegada de casi 6 mil cubanos a El Salvador, por vía aérea, donde siguieron por tierra rumbo a los Estados Unidos. En ese momento Costa Rica vivió lo que puede llamarse su "primera crisis migratoria" y desde entonces, Nicaragua mantiene sus fronteras cerradas para los migrantes indocumentados, y el gobierno de Costa Rica ha tenido que aumentar la atención más inmediata hacia estas poblaciones.

En el año 2016, enterados de la oleada de cubanos que empezó a recorrer el continente rumbo a los Estados Unidos, una nueva oleada de migrantes haitianos decidió emprender la ruta hacia este destino antes de la llegada de Donald Trump al poder para así intentar ingresar al territorio estadounidense antes de que se llevaran a cabo los cambios en las tan temidas políticas migratorias. En el 2016, con el arribo de la oleada de migrantes haitianos se da la segunda crisis migratoria, cuando miles de migrantes empezaron a quedarse bloqueados en la frontera con Nicaragua. Se instalaron entonces, en varias zonas fronterizas, centros de atención para los migrantes. Tuvimos la oportunidad de trabajar durante cinco meses en estos centros y fue en estos centros que realizamos la mayoría de las observaciones y entrevistas que sirven como datos empíricos para los análisis acá presentados.

\section{La ruta migratoria haitiana}

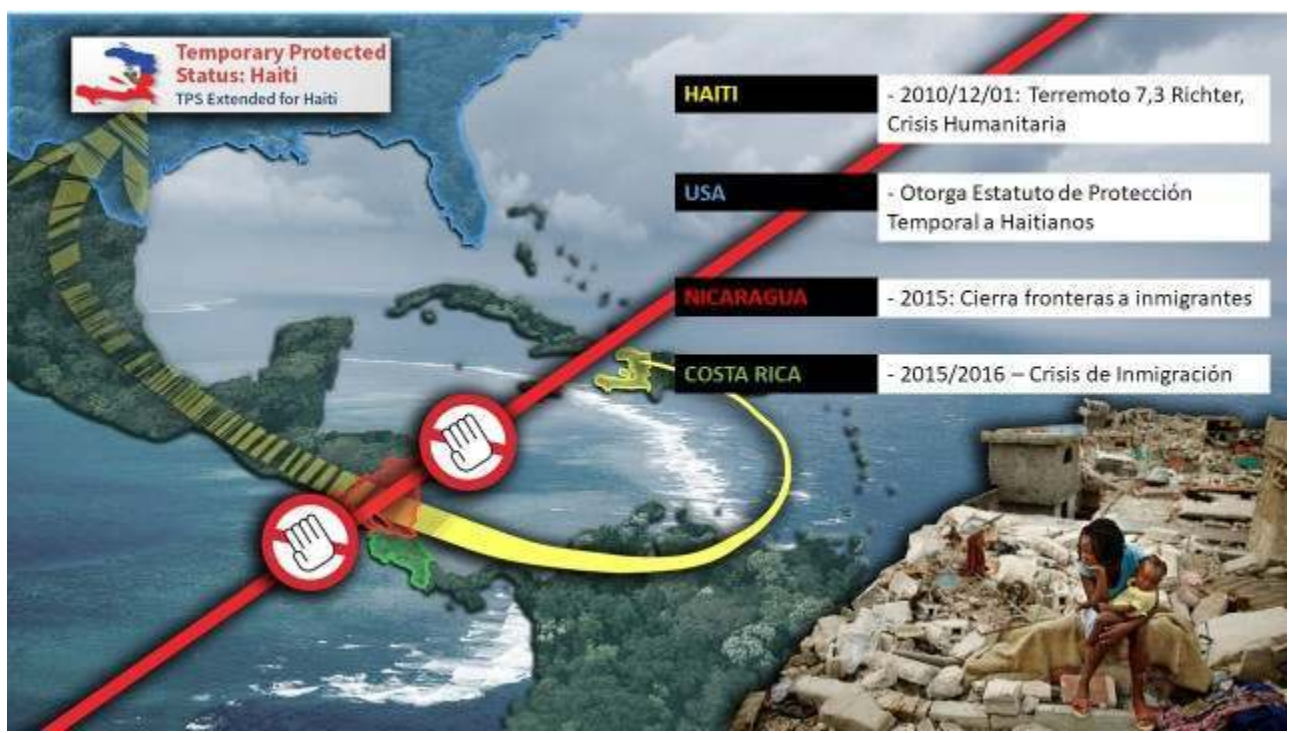

\section{La migración bajo el lente de las ciencias sociales}

La migración internacional ha sido abordada por las ciencias sociales desde una multitud de perspectivas y dimensiones, lo que por supuesto implica maneras distintas de 
aprehender el fenómeno. Múltiples son también los motivos que la generan. Nos interesaremos aquí más específicamente en el fenómeno migratorio desde el estudio de la experiencia de vida de los migrantes. En esta vía retomamos los análisis que presenta Abdemalek Sayad sobre la experiencia migratoria en donde habla del migrante como una persona que vive en una "doble ausencia" tanto en la sociedad de acogida como en la de origen. Son muchos los discursos que mencionan al migrante como un personaje desraizado pero estos conceptos se vuelven obsoletos cuando observamos la emergencia de nuevos espacios migratorios atravesados por las NTICS, caracterizados por la "presencia virtual". La "doble ausencia" queda desplazada por nuevas generaciones que se instalan en la movilidad y están dotadas de capacidades excepcionales para estar actualizando permanentemente sus vínculos con sus sociedades de origen y países de destino.

Encontramos también en la literatura sobre las migraciones, muchos estudios centrados sobre la organización de las redes de migrantes pero muy pocas de ellos tienen en cuenta las capacidades de los migrantes para apropiarse de las redes en las que están inmersos. Para esto creemos que es necesario sobrepasar las dicotomías más comunes cuando nos referimos a los migrantes de tipo móvil/inmóvil, ni aquí/ni allá, nómada/sedentario, etc., y priorizar en los desplazamientos de las personas, para no caer así en simplificaciones históricas sociológicamente obsoletas. Estas concepciones difícilmente tienen en cuenta la idea de un mundo en donde la movilidad se ha ido generalizando al mismo tiempo que se lleva a cabo una complejización sin precedentes de la comunicación.

En este punto, el enfoque dicho de las "capacidades" que plantea Amartya Sen nos parece interesante para abordar el fenómeno migratorio, en donde se parte de la noción de "bienestar" desde un ángulo distinto al que propone la visión economista "clásica" que reduce este concepto a su única dimensión monetaria o a la simple posesión de bienes materiales. El autor insiste sobre el hecho que el desarrollo humano es un fin en sí, mientras que el crecimiento económico no es a lo sumo más que un medio para acceder a este fin. El concepto de "capacidades" se vuelve central en los análisis de Sen, ya que él define estas capacidades como la libertad de poder escoger la vida que uno desea llevar. En el caso de los migrantes, este concepto se vuelve interesante cuando nos enfocamos en la decisión personal de desplazarse en busca de mejores condiciones de vida como motivo principal del desplazamiento.

18 Si bien proponemos abordar el fenómeno migratorio desde una óptica que puede considerarse de cualitativa, creemos que es necesario hacerlo situando a su vez al migrante en un sistema global que se inscribe en la lógica del "paradigma de la movilidad" tal como lo planteó el autor Alain Tarrius a finales de los años ochenta. Sin embargo, a este paradigma hay que agregarle el análisis del impacto de la fusión del uso las NTICS y la migración. Para el autor es necesario considerar al migrante en la totalidad de sus movilidades (ya sean físicas, imaginarias o virtuales) lo que nos llevará a verificar el pasaje de un tipo de sociedades en donde el "sedentarismo" era el estado dominante a sociedades en donde la "hipermovilidad" aparece como elemento central.

\section{La cultura del vínculo en la movilidad}

Cuando conjugamos los distintos planos a los que nos referimos anteriormente, vemos aparecer una cultura de la migración cuyo eje central es el vínculo que los migrantes crean y mantienen durante toda su movilidad con sus distintos grupos de pertenencia. Esta 
concepción, si bien está latente en todos los grupos sociales, se ha vuelto cada vez más visible y dinámica, una vez que los migrantes empezaron a utilizar masivamente las NTICS. Hoy en día resulta imposible pensar al migrante como una persona divida entre varios mundos y vemos como se perfila una definición que, si bien se basa en algunas formas de ruptura, aparecen la movilidad y la conectividad como nuevos elementos claves para entender estos procesos.

Retomamos acá los análisis de Dana Diminescu en su obra "El Migrante conectado. Por un manifiesto epistemológico" en donde la autora nos dice que la movilidad y la conectividad se volvieron los vectores que permiten asegurar y guiar las líneas de continuidad en la vida de los migrantes, así como en las relaciones que estos mantienen con las sociedades que transitan y a las que pertenecen. Para la autora lo que ayer se consideraba en la migración como un corte con las raíces hoy se ha vuelto sinónimo de circulación y mantenimiento de los contactos. Esta evolución estaría marcando una nueva era en la historia de las migraciones : la era de lo que la autora llama del "migrante conectado".

21 Es a partir de estas líneas de continuidad, evolución y vinculación, que nos planteamos abordar y comprender esta figura del migrante conectado. Las distintas teorías de la globalización, de las redes y los procesos transnacionales han puesto en evidencia algunos de los aspectos de esta nueva categoría de migrante como la multipertenencia (tanto a territorios como a redes), la hipermovilidad, la flexibilidad laboral, las capacidades de transformación y apropiación de los NTICS o las capacidades para convertir sus realidades de vida por otras más favorables.

\section{Estudios de caso : El desarrollo de estrategias innovadoras en materia migratoria por parte de la población haitiana en Costa Rica}

Durante el año 2016, ante la situación de bloqueo de las fronteras entre Nicaragua y Costa Rica, se contabilizó el paso de más de 40.000 personas de posible origen haitiano por suelo costarricense. Todos los migrantes llegaban indocumentados y se encontraban bloqueados en las fronteras de Costa Rica. El ingreso se realizó por la frontera sur del país y en este punto se les daba un papel migratorio de identidad para circular por el territorio costarricense, en donde, en base "a la palabra", las personas daban sus datos personales, sin ninguna forma de poder corroborar la veracidad de estos. Este número es bastante aproximativo ya que los mismos datos oficiales se contradecían constantemente en las cifras anunciadas.

Al analizar en detalle las noticias en los medios de prensa notamos una gran confusión entre las cifras mencionadas y los orígenes citados. (Ver anexos sobre tratamiento mediático del fenómeno migratorio por los principales medios de prensa costarricenses y nicaragüenses). Las noticias hablaban de miles de personas provenientes de África, algunas veces mencionaban que estas personas venían del Congo (sin especificar siquiera a cuál de los dos países llamados "República Democrática del Congo" o "República del Congo" pertenecían). El poco conocimiento de las sociedades africanas en la sociedad costarricense fue otro factor que facilitó la profusión y confusión de la información y el mal manejo de los datos colocando casi siempre a todos estos migrantes bajo la categoría simplista de "migrantes africanos". 

norte con Nicaragua, nos llamó de inmediato la atención el hecho de que prácticamente ninguna de las personas que ahí se encontraban, salvo algunas excepciones, venían de alguna nación africana y todos eran en su mayoría personas de nacionalidad haitiana. Venidos de varios rincones del continente americano (sus procesos migratorios habían empezado en la mayoría de los casos en otros países del cono sur, pasados años atrás) querían llegar a los Estados Unidos antes de que ocurriera el cambio de gobierno en enero del 2017. Durante cinco meses en los que se realizaron gran cantidad de entrevistas y observaciones, los migrantes afirmaron siempre haber recibido información vía sus redes sociales, a través de la plataforma de "Whatsapp", dónde se les indicaba que se declararan de nacionalidad congolesa con el fin de complicar los eventuales procesos de deportación, esto al no haber representación gubernamental de ese país en los países recorridos. El resultado de todo esto fue el de un clima de confusión e ignorancia acerca del verdadero origen de estas personas indocumentadas, empezando por el hecho de que no existe un país llamado "Congo" que fue el nombre bajo el cual ingresaron todas estas personas al territorio costarricense.

Existe aún campo para investigar a profundidad las distintas circunstancias que hicieron posible un tal estado de confusión en la sociedad costarricense con respecto a los miles de migrantes que estaban llegando al país, pero consideramos haber mencionado ya algunos puntos que nos parecen claves. Lo que hemos observado es como el teléfono móvil permitió a los migrantes llevar a cabo una apropiación de la tecnología para desarrollar sus propias estrategias en políticas migratorias y poder realizar sus recorridos de la mejor manera posible. Es decir que la "capacidad de conversión" de la realidad que mencionamos anteriormente, tal como la propone Amartya Sen, encuentra aquí un terreno fértil para analizar los usos de las tecnologías desde un lado más humano e incluyente y nos da pistas muy interesantes para continuar nuestras investigaciones.

Podemos ver a través de este estudio de caso como la variable comunicacional que brinda la telefonía móvil marca una ruptura en la historia de las migraciones. La tecnología móvil se volvió la herramienta clave para muchas personas que se instalan en la movilidad y que adquieren, gracias a esta, muchas capacidades para actualizar de manera permanente tanto sus redes personales, así como con los espacios que van transitando.

Cabe mencionar que los análisis que aquí proponemos forman parte de una investigación en curso sobre estos flujos migratorios que se encuentra aún en proceso, de ahí que señalamos las posibles pistas de investigación por las que nos gustaría continuar nuestro trabajo y no resultados definitivos de una investigación como tal. Nuestro análisis no pretende conceptualizar el fenómeno social de la migración sino que inscribe en una tendencia sociológica que pone en el centro del debate las capacidades estratégicas de los migrantes para instalare en un mundo global, poniendo en evidencia las relaciones existentes entre el desarrollo tecnológico de las técnicas de comunicación y las nuevas practicas migratorias.

\section{Conclusiones}

El auge de las NTICS en nuestras sociedades contemporáneas ha traído consigo cambios estructurales en muchos ámbitos que es necesario estudiar y cuestionar. El caso de la población migrante es un buen ejemplo y nos deja entrever pistas interesantes de 
reflexión para desarrollar nuevos enfoques que tengan en cuenta estas imbricaciones tecnológicas y su impacto en la vida de estas personas.

La "ruptura" que tanto caracterizaba los procesos migratorios se basaba en la precariedad de los antiguos medios de telecomunicación, así como en los altos costos de estos y se consideraba como el acto fundador de la migración al separar a las personas de su país de origen para situarlos en los países de acogida. Actualmente vemos como la situación cambió y asistimos a un proceso de densificación y multiplicación de las interconexiones transnacionales que van de la mano con los avances tecnológicos de los medios de comunicación y transporte. Podemos decir que la emergencia de Internet no solo vino a multiplicar los intercambios entre los migrantes, sino que modificó la naturaleza de estas interacciones y ofrece nuevas formas de organización de los tejidos sociales y simbólicos. Instalados en la movilidad, la posibilidad de mantener un contacto de forma regular con el país de origen (considerándolo no solo como un territorio físico sino también como un objeto simbólico) crea una figura del migrante que no calza con las definiciones antes utilizadas en los estudios de las migraciones. Las NTICS tienen su impacto en las practicas migratorias y ofrecen el marco para una redefinición de nociones tales como la identidad y la comunidad al tiempo que contribuyen a la multiplicación de posibilidades en la migración. Aquí el concepto de "comunicación transnacional" se vuelve clave para entender estos procesos y se refiere no solo a la comunicación interpersonal entre familiares y amistades que pueden estar en países distintos sino también a la búsqueda y mantenimiento de la relación con los referentes procedentes de las culturas de origen.

31 A lo largo de nuestra investigación nos pareció fundamental observar cómo los y las migrantes haitianos desarrollan múltiples estrategias para hacer, de los lugares donde llegan, un espacio de encuentro y apoyo. En nuestro trabajo se constató que los migrantes tienen la capacidad de apropiarse de las tecnologías para desarrollar sus propias estrategias y usos para así poder crear nuevas modalidades de interacción que les permitan avanzar en sus proyectos de vida. Es así como gracias a un dispositivo como el “ WhatsApp" miles de migrantes pudieron burlar las leyes migratorias de Costa Rica y poder seguir su camino hasta los Estados Unidos. Nos parece fundamental la necesidad de abordar estos fenómenos desde nuevas ópticas para elucidar pistas que produzcan futuras investigaciones en un campo que cada vez más se vuelve característico de los tiempos de hipermovilidad en los que estamos viviendo.

La migración haitiana representa además un ejemplo ilustrativo de los nuevos flujos multiformes y multicausales que reconfiguran los patrones migratorios continentales. Este nuevo flujo haitiano que recorre el continente americano, ciertamente, desafía las perspectivas analíticas y los abordajes políticos de la migración que se han venido teniendo desde las ciencias sociales y que no pareció urgente empezar a abordar. A pesar de la incertidumbre e inseguridad propias a los procesos migratorios, creemos que las NTICS permiten a los migrantes redefinirse conservando los elementos que pertenecen a su historia tanto individual como colectiva. Conservando su pasado pero marcados con el sueño del futuro, los migrantes encuentran en estos medios una estabilidad en medio de tantos cambios durante sus trayecto 


\section{BIBLIOGRAFÍA}

Álvarez Velasco, S. "Migración indocumentada en tránsito : la cara oculta de los procesos migratorios contemporáneos", en Serie Documentos de Trabajo, Documento No 10, CLACSO, Buenos Aires, 2001.

Appaduraí Arjun. Modernity at Large. Cultural Dimensions of Globalizatio. Minneapolis, University of Minnesota Press, 1996, 230 p.

BECK Ulrich. What is Globalization ?. Cambridge, Polity Press, (2000), 180 p.

Benítez, J. L. La comunicación transnacional de la e-familias migrantes. San Salvador : Programa de las Naciones Unidas para el Desarrollo (PNUD), 2011.

Bernal, G. « La migración haitiana hacia Brasil : Ecuador, país de tránsito”, en OIM (Ed.). La migración haitiana hacia Brasil : características, oportunidades y desafíos. Cuadernos Migratorios $\mathrm{N}^{\circ} 6$ (pp. 67-82). Buenos Aires : OIM - Oficina Regional para América del Sur, 2014.

Burbano, M. "Las asociaciones de migrantes haitianos en el Ecuador : entre debilidad y resistencia”. Revista Interdisciplinar da Mobilidade Humana - REMHU, vol. XXIII, nํ4, pp. 207-220, 2015.

Castles, S. La política internacional de la migración forzada. Migración y Desarrollo. No. 1. Octubre 2003, pp. 1-28. Ceja, I. "Migraciones haitianas en la región andina".

FLACSO - Boletín Andina Migrante, n. ${ }^{\circ}$ 19, pp. 2-13, 2015.

Bourjolly, J.M., « Haïti : quelle reconstruction? » en Buteau, Pierre ; Saint-Éloi, Rodney ; Trouillot Lyonel (eds.) Refonder Haiti? Mémoire d'encrier. pp. 25-32., Port au-Prince, 2010.

Brutus, É. y Chalmers C. « Construire ou reconstruire Haïti ? Acteurs, enjeux et Representations" en Buteau, Pierre ; Saint-Éloi, Rodney ; Trouillot Lyonel (eds.) Refonder Haiti ? Mémoire d'encrier. pp. 33-45. Port-au-Prince, 2010.

Canales, A. y Zlolniski, C..”Comunidades Transnacionales y Migración en la Era de la Globalización". Ponencia presentada en el Simposio sobre migración internacional en las Américas. San José, Costa Rica, 4 al 6 de Septiembre de 2000.

Castells Manuel. La société en réseaux. Tome I. L'ère de l'information, Paris, Fayard, 613 p, 1992.

Diminescu Dana “Le migrant connecté. Pour un manifeste épistémologique", Migrations/ Société, 17 (102), pp. 275-292, 2015.

Grau, M. I. La revolución negra, la rebelión de los esclavos en Haití 1791-1804.,Ocean Sur. San Salvador, 2015.

Jouet Josiane “ Relecture de la société de l'information", in Pierre Chambat, Communication et lien social, Paris, Descartes, pp. 177-190, 1992.

Mattelart Tristan “Les diasporas à l'heure des technologies de l'information et de la communication : petit état des saviors”, Tic\&société, 3 (1-2), pp. 11-57, 2010.

Neldecu Mihaela “ L'instrumentalisation de l'espace virtuel par une communauté de professionnels immigrés. Vers une nouvelle forme d'organisation diasporique ?," Autrepart, numéro spécial « Diasporas, développements et mondialisation », 22, pp. 147-165, 2002. 
Nedelcu Mihaela «(Re)penser le transnationalisme et l'intégration à l'ère du numérique. Vers un tournant cosmopolitique dans l'étude des migrations internationales?, » Revue Européenne des Migrations Internationales, vol. 26, $\mathrm{n}^{\circ}$ 2, pp. 33-55, 2010.

OIM. Glosario sobre Migración. Ginebra : Organización Internacional para las Migraciones, OIM, 2006. Robeyns, I. “The capability approach : A theoretical survey”. Journal of Human Develop, 2005. Sandoval García Carlos. No más muros. Exclusión y migración forzada en Centroamérica, San José : UCR, 2015.

Sayad Abdelmayek, La double absence. Des illusions de l'émigré aux souffrances de l'immigré, Paris, Seuil, 438 p, 1999.

Sen, A.K. "Development as freedom". Oxford University Press, 1999. Sen, A.K. “The Idea of Justice". Harvard University Press, 2009.

Tarrius Alain. Territoires circulatoires et espaces urbains, Les Annales de la Recherche Urbaine, 59-60, pp. 50-60, 1993.

Castles, S. y Miller, M. J. “La era de la migración Movimientos internacionales de población en el mundo moderno. Colección América Latina y el Nuevo Orden Mundial”, Miguel Ángel Porrua, UAZ, Cámara de Diputados LIX Legislatura, Fundación Colosio, Secretaría de Gobernación, Instituto Nacional de Migración, México, 2004.

Metzner, T. (2014). "La migración haitiana hacia Brasil : estudio en el país de origen”, en OIM (2014) "La migración haitiana hacia Brasil : Características, oportunidades y desafíos”, Cuadernos Migratorios ํㅜㅇ, OIM, Lima, pp. 15-32, 2014.

Nieto, C. Migración haitiana a Brasil. Redes migratorias y espacio social transnacional, CLACSO, Buenos Aires, 2014.

Vásquez, T ; Busse, E ; Izaguirre, L. “La migración haitiana en Perú y su tránsito hacia Brasil”, en "La migración haitiana hacia Brasil : Características, oportunidades y desafíos", Cuadernos Migratorios ํํㄴ, OIM, Lima, pp. 83-106, 2014.

\section{ANEXOS}

Anexo 1. Principales flujos migratorios en el Continente Americano, elaborado por la Organización Internacional para las Migraciones (OIM).

Fuente : http://www.iom.int/jahia/webdav/shared/shared/mainsite/ policy_and_research/un/65/A_65_203_S.pdf

Anexo 2. Capturas de pantalla de varios medios de prensa 
NACIONAL

Pais atencio el trănito ce 26.000 personas en poco más de un año

Migrantes de paso en Costa Rica superaron la población de La Cruz, el principal refugio

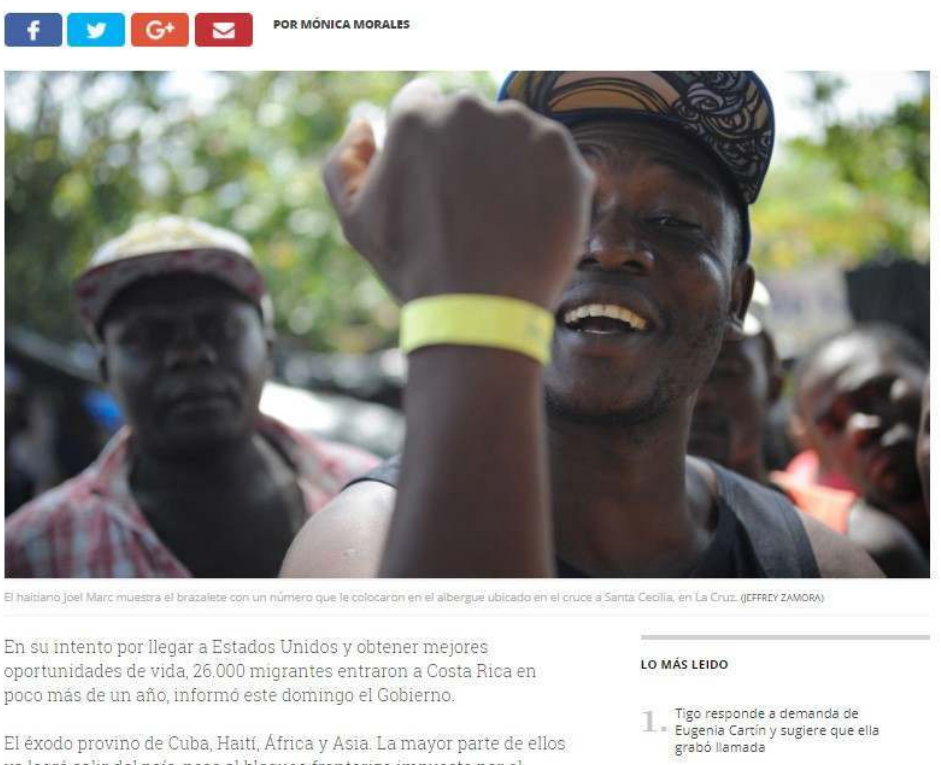

Sléxodo provino de Cuba Haiti Africa y Asia La mayor parte de ellos

- Eugenia Cartin y sugiere que el

Fuentes : La Nación(

http://www.nacion.com), Crhoy (https://www.crhoy.com), La Prensa (http:// www.laprensa.com.ni) y Univision noticias (http://www.univision.com/noticias)

4 univisionNOTICIAS

\begin{abstract}
Los migrantes olvidados: miles de africanos permanecen varados en Costa Rica

En una nueva ruta migratoria, miles de africanos han cruzado el Atlántico en los últimos meses con el fin de llegar por tierra a EEUU. Pero la crisis de inmigrantes cubanos en Centroamérica los dejó atrapados en Costa Rica. Univision Noticias estuvo en un campamento donde medio millar de ellos permanecen refugiados.

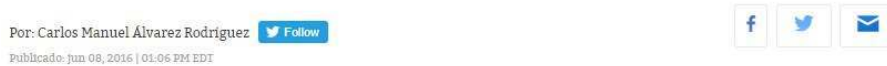

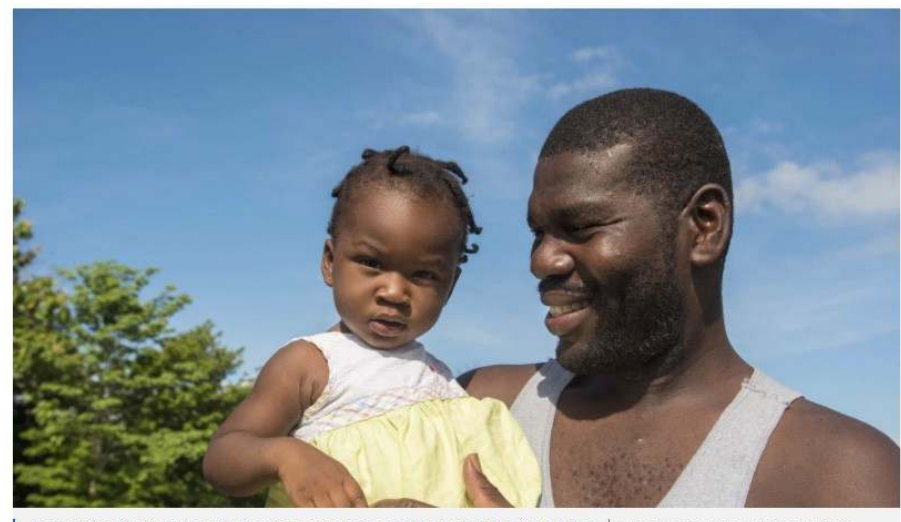

| La mayoria de inmigrantes africanos de Paso Canoas no quiere revelar su pais de origen. | Eyleen Vargas para Univision Noticlas 


\section{LA PRENSA /NACIONALES}

\section{Más de $\mathbf{2 0}$ mil migrantes africanos y haitianos ingresan a Costa Rica}
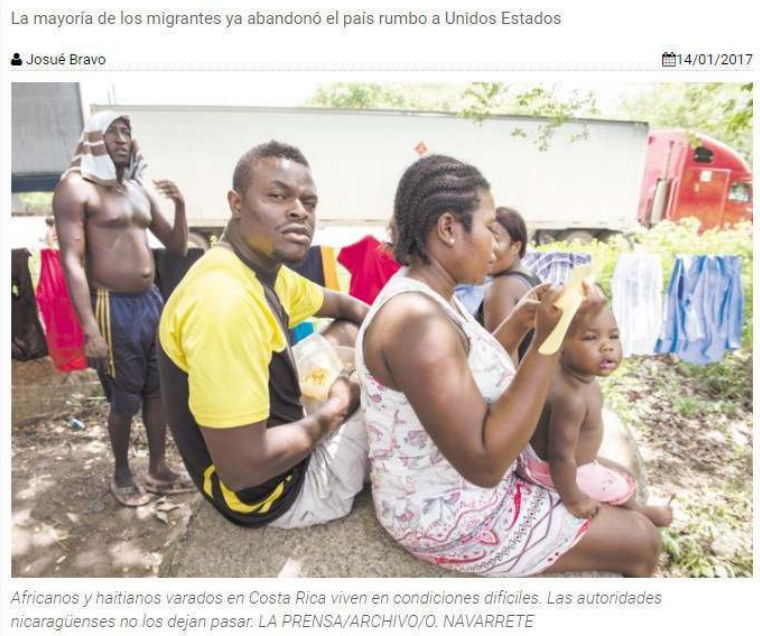

Costa Rica ha permitido el ingreso desde Panamá de 20,208 inmigrantes haitianos,

africanos y asiáticos desde el pasado 21 de abril, día en que este país inició una medida para salir de una crisis migratoria generada por la aglomeración de cientos de migrantes en su frontera sur, Paso Canoas

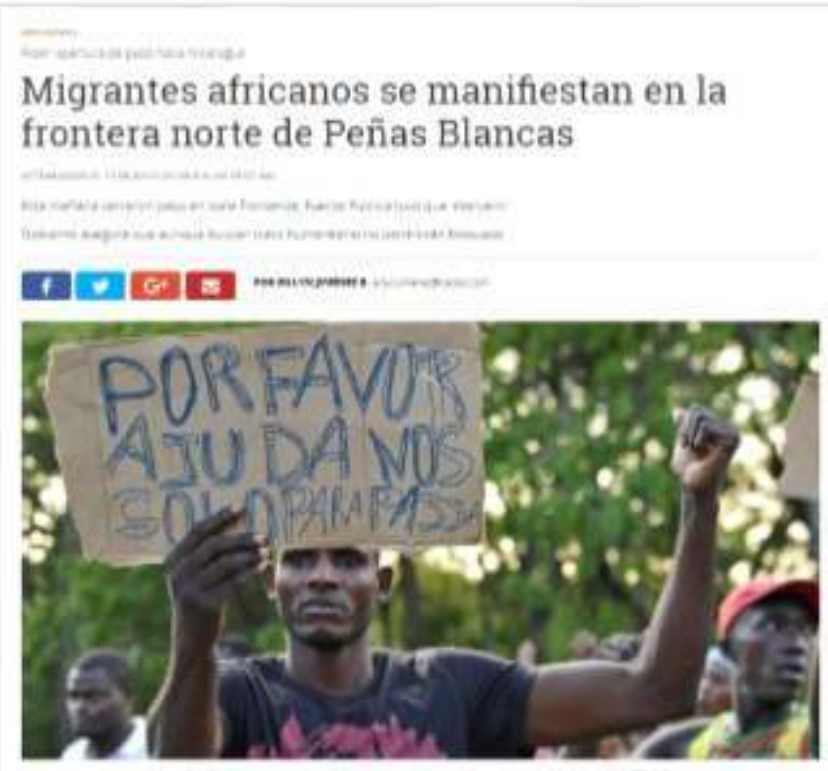

NOTAS

1. Sandoval García Carlos "No más muros. Exclusión y migración forzada en Centroamérica", San José, UCR, 2015.

2. https://datos.gob.mx/busca/dataset/instituto-nacional-de-migracion 


\section{RESÚMENES}

Los constantes progresos en materia de tecnologías móviles tienen un fuerte impacto sobre las actividades humanas de manera general y en el caso de las migraciones internacionales están forjando una serie de características particulares que se diferencian de los flujos migratorios en sus etapas anteriores. El reciente y acelerado aumento de la llegada de migrantes indocumentados de origen haitiano o africano al territorio costarricense nos permitió desarrollar una serie de reflexiones alrededor del impacto de las NTICS sobre estas poblaciones en sus experiencias migratorias, en situaciones en donde la intensa movilidad de las personas ha hecho del teléfono móvil un instrumento indispensable para llevar a cabo de buena manera sus desplazamientos a través del continente.

Les constants progrès en matière des technologies mobiles ont un fort impact sur les activités humaines de façon générale et dans le cas des migrations internationales ils sont en train de créer une série de caractéristiques particulières qui différencient ces flux migratoires des ses étapes antérieures. L'augmentation récente et accélérée de l'arrivée de migrants sans papiers de nationalité haïtienne et africains dans le territoire costaricien nous a permis de développer des réflexions autour du sujet de l'impact des NTICS sur ces populations dans son expérience migratoire, situation où l'intense mobilité des personnes fait du téléphone portable un instrument indispensable pour le bon accomplissement de leur déplacement à travers le continent.

The constant progress in matter of wireless technologies have a strong impact in all human activities in general, in the case of international migrations these technologies are forging a series of specific characteristics that differ from previous stages in migratory flows. The recent and accelerated rise of the arrival of Haitian or African origin undocumented Immigrants to Costa Rican territory, has allowed us to develop a series of reflections around the impact of the NICT'S on these populations and their migratory experiences, where the intense movement of these people have made mobile phones a crucial instrument to carry out properly their trips across the continent.

\section{ÍNDICE}

Keywords: migration, Haiti, migratory routes, central America, NICT, mobile telephony, globalization, virtuality, conversion, migratory strategies, transnational community, mobility Palabras claves: migración, Haití, rutasmigratorias, América central, NTIC, telefonía móvil, mundialización, virtualidad, conversión, estrategias migratorias, comunidades trasnacionales, movilidad

Mots-clés: migration, Haïti, routes migratoires, Amérique centrale, NTIC, téléphonie mobile, mondialisation, virtualité, conversion, stratégies migratoires, communautés transnationales, mobilité 
AUTOR

DANIELA CASTRO CABALCETA

Investigadora independiente y colaboradora con la Cooperativa Sulá Batsú, Costa Rica 